\title{
De la frase nominal a la frase determinativa
}

\author{
JORGE ESQUIVEL VILLAFANA \\ Departamento Académico de Lingüística
}

IF n el desarrollo de la lingüística moderna, resulta evidente la extraordinaria

relevancia que ha experimentado la categoría lexical determinante en desmedro de los otros constituyentes de la FN en la consideración de su status jerárquico, pues ha pasado de ser un elemento no nuclear (opcional u obligatorio) en la estructuración nominal en la gramática generativa (según Chomsky, Postal, Fillmore, etc.) a una posición nuclear, generadora de su propia proyección máxima, la frase determinativa, en la gramática categorial (según Abney).

A través de los años, desde 1957 a la actualidad, su posición ha ido consolidándose, según lo refieren las diversas versiones de FN que fueron configurándose con el soporte de la evidencia linguística del momento. De aquí nuestro interés de examinar su impresionante evolución en el marco de las dos corrientes modernas: la generativay la categorial El análisis delas versiones de Chomsky, Postal, Fillmore, y también da de Abriey, como los más destacados representantes de ambas gramáticas, nos permitirá conocer no sólo las motivaciones teóricas que sustentaron las versiones en cuestión, sino también la evolución y el enriquecimiento de los argumentos teóricos en favor de la propuesta de Abney, que se asume como la más adecuada en la actualidad.

\section{Introducción}

Dentro de los parámetros de la ciencia, es difícil hablar de verdades absolutas, por lo que ninguna disciplina o corriente científica se puede jactar de poseer las llaves para descubrirlas; a lo más, lo que podemos encontrar son propuestas, conjeturas, hipótesis generadas para dar cuenta de los hechos científicos. El problema que se plantea ante la pluralidad de las propuestas es decidir cuál de ellas resulta descriptivamente la más adecuada, la que cobra mayor significación como para considerarla en desmedro de las otras. 
En el marco del pensamiento chomskyano, las propuestas sobre los constituyentes de la FN y el status jerárquico de ellos ha ido variando en el tiempo desde una propuesta casi tradicional, en sus primeras versiones, hasta la moderna y revolucionaria de Abney, ofrecida desde la teoría de Principios y Parámetros. El mismo Chomsky, en "Aspectos..." reclama la necesidad de un artificio que permita valorar las hipótesis en juego y que permita elegir la mejor opción, la llama a ésta "capacidad valorativa de hipótesis". Según ésta, la mejor opción es aquella que se adecua mejor al fenómeno de los datos; es decir aquélla cuyos hechos la confirman como la más adecuada para el tratamiento de los fenómenos lingüísticos. En realidad, Chomsky reclama que se evalúe los méritos de las formulaciones gramaticales rivales de modo que se pueda elegir la que capture mejor la capacidad intuitiva del hablante, la que explique mejor las regularidades subyacentes del lenguaje. En este intento, el análisis de las evidencias lingüísticas que soporten la propuesta más adecuada acerca de la estructura de la FN, en el sentido de si el status del constituyente determinante resulta opcional $\mathrm{u}$ obligatorio, o si se constituye en el núcleo de su propia proyección, la FD, nos lleva a poner en juego la consideración de las hipótesis establecidas diacrónicamente.

\section{Noam Chomsky}

Syntactic Structures representa una de las versiones más tempranas de Chomsky. Publicada en 1957 , tỉente todavia la influtenciade la corriente estructuralista que se proponía superarpperedifiere de ella encque en lugar de darle a las oraciones de una lengua una descripción jerárquica adecuada, en el sentido de representar las relaciones entre cada uno de los constituyentes de la oración, enfatiza el carácter generativo de su teoría gramatical, lo que lo lleva a proponer reglas gramaticales en una forma radicalmente novedosa que lograrían generar sólo las oraciones correctas de una lengua. Plantea un sistema compuesto de tres partes: a) reglas de estructura frasal, b) reglas transformacionales y c) reglas morfofonémicas.

En este sistema, la oración es definida como una construcción conformada por frase nominal y frase verbal:

\section{(1) $\mathrm{O} \rightarrow \mathrm{FN}+\mathrm{FV}$}

Aunque no da definiciones válidas de FN y FV, pues presupone, al menos, que el concepto de ellas ha sido ya definido, se encarga de definir más bien el 
concepto de los constituyentes de ellas, ${ }^{1}$ pero desde una perspectiva de reglas de estructura frasal, encargadas de la reescritura de símbolos individuales a partir de símbolos que aparecen a la derecha, consideradas éstas desde una perspectiva superficial. La frase nominal es reescrita como:

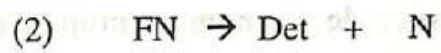

y la frase verbal como:

\section{(3) $\mathrm{FV} \rightarrow \mathrm{V}+\mathrm{FN}$}

Los números que van entre paréntesis y preceden cada orden de reescritura hacen referencia a las reglas de la gramática.

El determinante puede ser reescrito como 'el', 'la', 'los', 'las' o 'un', 'una', 'unos', 'unas' según el artículo que se elija para un nombre en determinado género o número. En tanto las características morfológicas del nombre determinan la forma de los elementos que to acompañan, la regla que lo especifica es denominada contextual.

Los términos frase nominal y frase verbal son usados por Chomsky como categorías sintagmáticas formales, vacías de contenido, por lo que no se llega a establecer el status jerárquico de los componientes de la FN.

Es recién en Aspectos de la teoría de la sintaxis (1965), que Chomsky, al considerar la propuesta de la existencia de la estructura profunda en la oración, ofrece una propuesta acerca de la estructura básica de la FN, la cual la conforman el N(ombre), obligatoriamente; el Det(erminante) y la O(ración), opcionalmente.

\section{(4) $\quad \mathrm{FN} \rightarrow$ (Det) $\mathrm{N}(\mathrm{O})$}

Esta regla, explicada en detalle, establece cuatro posibles estructuras de la FN:
a) $\mathrm{FN} \rightarrow$
$\mathrm{N}$

t. "Notice that general significance of the definitions depends on the asssumption that the symbols S, NP, VP, $\mathrm{N}$ and $\mathrm{V}$ have been characterized as grammaticals universals". En Ch. Rohrer (1978: 87). 
b) $\mathrm{FN} \rightarrow$ Det $+\mathrm{N}$
c) $\mathrm{FN} \rightarrow \mathrm{N}+\mathrm{O}$
d) $\mathrm{FN} \rightarrow$ Det $+\mathrm{N}+\mathrm{O}$

a) describe la ocurrencia de FN conformada por el N solamente, cuando éste tiene la característica de ser nombre propio, por lo que carece de determinante alguno;

b) describe una estructura en la que aparece $\mathrm{N}$ precedido de Det, lo que ocurre cuando el nombre es común;

c) se refiere al nombre modificado por oración (o cláusula subordinada);

d) describe la clase de nombres que toman oraciones complementarias.

De hecho, esta propuesta que evidencia el carácter opcional del determinante fue, por entonces, la más reconocida y utilizada en los textos de gramática generativa de carácter divulgatorio.

\section{Paul Postal}

La propuesta ofrecida por Postal (1966) asume, por el contrario, que el determinante no es constituyente de la FN en la estructura profunda. Los posibles constituyentes de la FN los expresa mediante la siguiente regla:

\section{$\mathrm{FN} \rightarrow \mathrm{N}$ BP)lioteca de Letras}

Según esta regla, sólo hay dos posibles estructuras de constituyentes de la FN:
a) $\mathrm{FN} \rightarrow \mathrm{N}$
b) $\mathrm{FN} \rightarrow \mathrm{N}+\mathrm{O}$

a) describe el caso de una FN conformada por sólo el nombre;

b) expresa el hecho de una FN conformada por el nombre y oración.

Postal considera en su propuesta que los determinantes y pronombres son categorías estrechamente relacionadas que aparecen expresados en la estructura profunda como rasgos de nombres. Esto es, una regla de segmentación opera sobre todos los nombres para crear cualquiera, un determinante o un pronombre. ${ }^{2}$

2 Recuérdese el hecho de que el determinante y el pronombre aparecen en distribución complementaria en la estructuración de la FN; así como también que, en muchos casos, el proceso de pronominalización es visto como la deleción del nombre de la FN, excepto el determinante, el cual adquiere, entonces, el rótulo de pronombre. 
Si el nombre no está marcado pronombre, un determinante es insertado: el, la, los, las si el nombre es definido; un, una, unos, unas en otros casos. Si el nombre está marcado pronombre, un pronombre es insertado: él, ello, etc. si el nombre es definido; unos, unas, en otros casos. Finalmente, una deleción elimina el nombre original de la oración, puesto que el pronombre ha sido insertado.

\section{Charles Fillmore}

A diferencia de las propuestas anteriormente expuestas, Fillmore (1967) asume más bien que es el determinante el único constituyente obligatorio en la estructura de la FN, y que las categorías de N(ombre) y O(ración) (o cláusula subordinada) son opcionales en su conformación, según lo expresa en la regla (6):

(6) $\mathrm{FN} \rightarrow$ Det $(\mathrm{N})(\mathrm{Q})$

La lectura de esta regla nos permite reconocer tres posibles estructuras de FN:

a) $\mathrm{FN} \rightarrow$ Det $+\overrightarrow{\mathrm{N}}$

b) $\mathrm{FN} \rightarrow$ Det $+\mathrm{O}$

c) $\mathrm{FN} \rightarrow$ Det BibliNtect deoetras

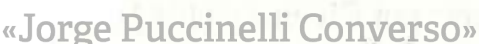

en las que sólo el determinante aparece como el elemento reiterativo de ellas.

Para sustentar esta propuesta, apela a la descripción del proceso de transformación denominado "pronominalización". Según éste, la pronominalización de la FN es entendida como el resultado de la deleción del nombre, menos el determinante, el cual es entonces llamado pronombre:
a. María trajo la tiza
b. José limpió los muebles
María la trajo
José los limpió

Para el caso de la pronominalización de las oraciones complementarias (específicamente aquellas que cumplen la función de objeto directo del verbo de la cláusula principal), se asume la expansión de la FN subcategorizada al verbo como Det y $\mathrm{O}$ (ración), de modo tal que al producirse la pronominalización de oración se delee ésta y el determinante se transforma en la forma pronominal 
lo, que -en tanto neutra- es la única forma pronominal posible, pues las oraciones en sí carecen de género y número:

(8) a. Juan sabe que María aprobó el curso

b. Yo dudo que cantes bien

Juan lo sabe. Yo lo dudo.

Tal proceso sería inviable sin la presencia del Det(erminante) en la expansión de la FN, de ahí que Fillmore considere su obligatoriedad en la estructura profunda de la FN.

Hasta aquí las propuestas analizadas. Mientras Postal niega la presencia del Det en la estructura profunda de la FN, Chomsky y Fillmore coinciden en considerarla como constituyente de ella. La diferencia entre estos dos últimos reside en el tratamiento que le dispensan, pues mientras Chomsky lo propone como opcional en la estructuración de la FN, Fillmore lo asume como elemento obligatorio.

4. S. Abney

La más novedosa propuesta con respecto al tratamiento del determinante corresponde a la ofrecida por la gramática categorial en su versión teórica de Principios y Parámetros. Esta teoría es parte de la formulación propuesta por Chomsky (1970), la que fue desarrollada posteriormente por Jakendoff (1977) y reformulada nuevamente por Chomsky (1982).

"Jorge Puccinelii Converso"

Dentro del marco de esta teoría gramatical, se reconoce dos clases de categorías: lexicales y funcionales. El elemento diferencial entre ellas lo constituye el aspecto semántico. Las categorías lexicales lo conforman aquellas palabras que tienen significado, contenido descriptivo. Tenemos en el español hasta cuatro categorías lexicales: N(ombre), V(erbo), A(djetivo) y $\mathrm{P}$ (reposición). Estas son definidas mediante el uso de rasgos distintivos:

(9) $\begin{array}{lllll}\mathrm{A} & = & +\mathrm{N} & +\mathrm{V} \\ \mathrm{N} & = & +\mathrm{N} & -\mathrm{V} \\ \mathrm{V} & = & -\mathrm{N} & , & +\mathrm{V} \\ \mathrm{P} & = & -\mathrm{N} & -\mathrm{V}\end{array}$

Así, el nombre es descrito, sintácticamente, con los rasgos de (+N, -V); el adjetivo, $(+\mathrm{N},+\mathrm{V})$; el verbo, $(-\mathrm{N},+\mathrm{V})$; la preposición, $(-\mathrm{N}, \quad-\mathrm{V})$. Las 
categorías funcionales son aquellas que tienen propiedades sintácticas pero que carecen de significado, son de naturaleza estrictamente formal: Compl, Flex, y Det. Las categorías léxicas se constituyen en el núcleo de una expansión o pro-yección máxima denominada sintagma (o frase). Cada núcleo aparece, entonces, acompañado de esp(ecificador) y compl(emento).

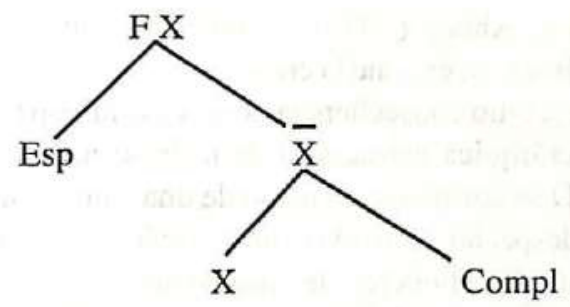

El nombre y el verbo constituyen las categorías lexicales centrales de la sintaxis en la medida que ellas representan los pilares de la estructura semántica de la oración: argumentos y predicados. El nombre es el ítem lexical con el que designamos seres y objetos del mundo real o imaginario. Aparece como el núcleo de una estructura cuya máxima proyección resulta la frase nominal, de ahí que se considere su constituyente obligatorio ya como categoría léxica, ya por medio de una entidad sintáctica desprovista de contenido. Tiene dos modificadores: los especificadoresc(losddeterninantes) y los complementos (los adjetivos), los que soge considerados estructuralmente opcionales. Los pronombres personales comparten la posibilidad de funcionar como núcleos de la FN, por lo que deben considerarse también como $(+\mathrm{N},-\mathrm{V})$. La categoría lexical determinante, especificador del nombre, depende directamente del nódulo FN y es el modificador más externo de la FN. (11)

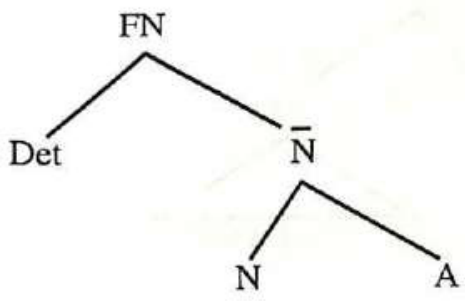


Tal presentación de la FN, que constituye la reformulación más moderna del pensamiento chomskyano, tiene la ventaja de expresar claramente la jerarquía de los constituyentes de la frase para lo cual se utiliza hasta tres niveles: proyección máxima, proyección intermedia y núcleo; esto, en desmedro de los modelos anteriores caracterizados, en ausencia de jerarquía, por la presentación lineal de sus constituyentes.

Sin embargo, sería S. Abney (1987), quien en el marco de esta misma teoría de Principios y Parámetros, expone la creación de la llamada frase determinativa (FD), hipótesis que, como consecuencia de una evolución teórica, revolucionaría la concepción jerárquica estructural de la frase nominal. Según esta propuesta, la categoría FD se construye en torno de una categoría funcional llamada Det(erminante). A despecho de lo que el marco teórico sugiere, la categoría funcional Det se constituye en el núcleo de una estructura independiente y da lugar a sus propias proyecciones donde la máxima proyección es conocida como frase determinativa. La FN se convierte en el complemento del núcleo (12 a). Este revolucionario modelo resquebraja la noción jerárquica que normalmente se establecía entre los constituyentes de la estructura nominal en los modelos de análisis anteriores a Abney (12 b). La primordial modificación de esta propuesta consiste en establecer un status diferente para la categoría Det(erminante), la que pasa de ser el especificador del núcleo Nombre en una estructuración nominal común para convertirse en el núcleo de la frase determinativa en la cual la proyección mâxima del nombre àctúa comoccomplemento.

\section{"Jorge Puccinelli Converso»}

a)

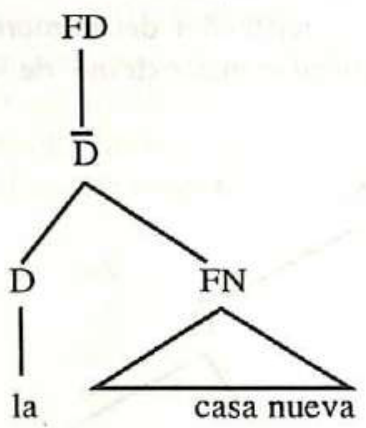


b)

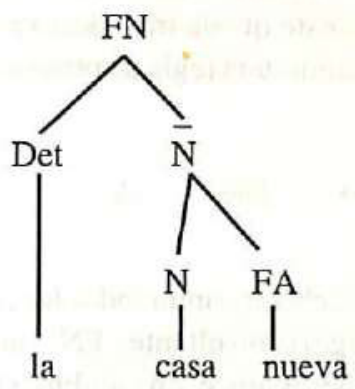

La hipótesis de $\mathrm{Abney}^{3}$ se fundamenta, básicamente, en las siguientes consideraciones:

Primera. El orden relativo entre el determinante y el nombre respeta el patrón "núcleo complemento" de otros casos de selección. La selección debe estar cifrada, pues, en la entrada léxica del determinante y no en la del nombre.

Segunda. El hecho de que ciertos determinantes, comó el artículo definido, no puedan aparecer independientemente, significa que seleccionan obligatoriamente un complemento. El fenómeno sería el mismo que ciertos verbos o ciertas preposiciones que exigen la presencia de un elemento que los complemente.

Tercera. El hecho de que haya determinantes que sí pueden aparecer con independencia del nombre y el que en tales casos asuman plenamente en el enunciado el papel de la estructura nominal completa significa que la categoría que cabe atribuir a este tipo de estructura ha de ser proyección de Det, no de N.

(14) Esa mujer no le conviene

[SD esa [FN mujer]] no le conviene.

${ }^{3}$ Para mayores detalles, véase Haegeman (1991, 1994), Fernández Lagunilla y Anula Rebollo (1995). 
Cuarta. Los determinantes también se combinan con adjetivos. En tales casos, se suele decir que éste queda traspuesto a la categoría nominal. Por tanto, habrá que asumir una regla de proyección categorial del siguiente tipo:

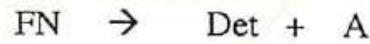

Esta regla, sin embargo, choca contra todas las posibilidades de desarrollo categorial, puesto que la categoría resultante-FN- no es ninguna de las categorías de partida. El problema desaparece, en cambio, si se admite que el determinante es el núcleo de esa estructura y que la proyección máxima es una proyección suya (FD):

$$
\mathrm{FD} \rightarrow \text { Det }+\mathrm{A}
$$

Recuérdese que, en el entorno generativista, una estructura como "ese pecoso" -compuesta por determinante y adjetivo- se analiza como el caso de un adjetivo (pecoso) que se traspone a la categoría nominal (nominalizado), de modo que el nódulo dominante resulta una $\mathrm{FN}$, lo cual atenta, incluso, contra el sentido común, pues el rótulo del nódulo frasal resultante es ajeno a sus constituyentes categoriales. En cambio, dentro de la hipótesis de Abney es permitido prescindir de este tipo de actitud teóriea pues, simplemente, el problema desaparece: la frase determinativa 'ese pecóso’" lés vista como una estructura compuesta por el Det 'ese' como núcleo; resulta obvio, por tanto, que la FD sea la máxima proyección del núcleo determinante.

\section{Conclusiones}

El presente trabajoofrece una exposición panorámica acerca de las principales hipótesis que se generaron con el intento de determinar la posible estructura y jerarquía de los constituyentes de la FN. Históricamente, ésta ha pasado de una presentación bastante vaga y de orden lineal hacia una de mayor precisión y de orden jerarquizado en la que la categoría de determinante ha cobrado relevancia impactante. Esta evolución hacia una estructura como la propuesta por S. Abney -que ha capturado la preferencia de los especialistas- se corresponde, por lo demás, con el patrón "núcleo-complemento" en términos de una gramática universal. 
Finalmente, queda por decir que la propuesta de este modelo afina aún más el análisis sintáctico en lo relativo a la moderna distribución jerárquica de los elementos tradicionalmente considerados en la estructura nominal.

\section{BIBLIOGRAFIA}

\section{CHOMSKY, Noam}

1957 Syntactic Structures. The Hague. Mouton.

1965 Aspects of the Theory of Syntax. MIT Press, Cambridge, Massachusetts.

1982 La nueva sintaxis.Teoría de la rección y el ligamiento. MIT. Ediciones Paidós Ibérica S.A., Barcelona.

DEMONTE, Violeta

1994

La teoría sintáctica: de las estructuras a la rección. Editorial Sintesis S.A. Madrid.

FERNÁNDEZ LAGUNILLA, M. y Alberto ANULA REBOLLO

1995

Sintaxis y cognición.Introducción al conocimiento, el procesamiento y los déficits sintácticos. Editorial Síntesis S.A., Madrid.

HAEGEMAN, Liliane

1991-1994 Introduction to Gobernment and Binding Theory. Blackwell Publishers.

HERNANZ, María Luisa y BRUCART, José María

1987 La sintaxis. Editorial Crítica S.A. Barcelona. 
LORENZO GONZALES, Guillermo

1996 Introducción a la Sintaxis Generativa. Alianza Editorial S.A., Madrid.

RIEMSDIJK, Henk Van y Edwin WILLIAMS

1986

Introducción a la teoría gramatical. Cátedra, Madrid.

ROHRER, Christian

1978

Lingüística Funcional y Gramática Transformativa. Editorial Gredos, Madrid.

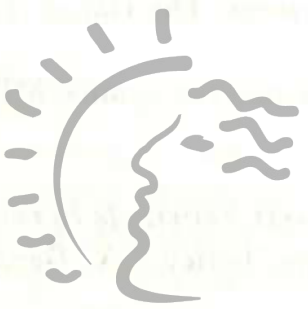

Biblioteca de Letras

"Jorge Puccinelli Converso» 